untried matings between parents having dissimilar characteristics. The more highly developed condition dominates over the less highly developed ; and in the extreme case the presence of a character dominates over its entire absence. This extreme case gives results that accord with Mendel's law; the cases in which the characteristics of the parents differ merely in degree are often not inherited precisely in accordance with that law.

\title{
$60(316)$
}

\section{The experimental production of the maternal part of the placenta in the rabbit. ${ }^{1}$}

\section{BY LEO LOEB.}

[From the Laboratory of Experimental Pathology, University of Pennsylvania.]

In a former communication ${ }^{2} \mathrm{I}$ stated that $\mathrm{I}$ had been able to produce at will, decidual tumors in the uterus of the guinea-pig by making deep incisions into the wall of the uterus, at certain periods of the sexual cycle. The nodules thus produced belong to that class of formations which I designated as "transitory tumors." Since then, further investigations ${ }^{3}$ have demonstrated that these deciduomata are produced under the influence of an internal secretion of the ovaries. There are indications that it is the corpus luteum which produces this internal secretion. If such a "preparing substance" has been secreted by the ovaries, indifferent stimuli are sufficient to call forth the development of the deciduomata. In the guinea-pig the new formations show a structure identical with that of the decidua. Large cells of connective tissue origin show an epithelial arrangement. The nuclei of many cells are hypertrophic, the cytoplasm is, on the whole, solid, and stains well with eosin. The decidual tissue of the placenta of the rabbit differs in some important respects from that of the guinea-pig, if we compare the placentas at the corresponding stages of development. In the rabbit the decidual cells are quite vacuolar and the

\footnotetext{
1 This investigation was carried out under a grant from the Rockefeller Institute for Medical Research.

2 Loeb: This journal, rgo7, iv, p. 93.

${ }^{3} \mathrm{~A}$ report on these further investigations appeared in the Journal of the American Medical Association, June, rgo8.
} 
blood vessels show an interesting change. At a very early period the endothelial lining becomes transformed into syncytial and plasmodial masses. It was of interest to determine whether the decidure produced experimentally in the guinea-pig and in the rabbit differed in the same way as the normal deciduæ in these two species.

The following experiments were, therefore, carried out:

I. In four female rabbits which had been kept separated from males for some time previous to the operation and which were not pregnant, no deciduomata were formed after the usual incisions into the uterus had been made. We found only edematous mucosa on microscopic examination.

2. Three rabbits were operated, 3 to 5 days after copulation. In two of these animals the nodules, formed ten days after the operation, were examined microscopically. They showed the above mentioned characters of the maternal part of the placenta of the rabbit.

3. These latter experiments were, perhaps, open to the objection that ova had come into contact with that part of the mucosa which gave rise to the formation of decidual tissue. Although such an occurrence was extremely unlikely inasmuch as the continuity of the uterine cavity had been interrupted, through the operation, at many places, two other experiments were performed, in which, in each case, both fallopian tubes were tied at their junction with the uterine horns about $13 / 4$ hours after copulation. Seven days later the ordinary incisions were made into the uterus. Ten days later many decidual nodules were formed in both cases, which, on microscopic examination, showed the characteristic features of the rabbit placenta, namely, the vacuolated cells, the plasmodial changes of the blood vessels, and besides, the formation of multinucleated cells in the uterine epithelial layer, which represented the first step in the formation of epithelial plasmodia.

These changes, however, did not occur at every point simultaneously. The plasmodial transformation of the blood vessels was sometimes seen without the accompanying formation of vacuolar cells; at other places both changes were present in the same area. On the whole, the nodules in the rabbit were, in proportion to the size of the rabbit uterus, considerably smaller than the deciduomata formed in the guinea-pig. 
From these investigations the conclusion can be drawn that the experimentally produced deciduomata differ in the rabbit and in the guinea-pig in the same way as the natural placentas do. Whether the dissimilar reactions of homologous tissues to similar stimuli in the rabbit and in the guinea-pig depend upon a difference in the structure of the protoplasm, or upon a quantitative or qualitative difference of the internal secretion of the ovaries, or upon a combination of these two factors, are questions which cannot be answered at present.

\section{I (3I7)}

Hemolytic action of the venom of Heloderma suspectum.

By ELIZABETH COOKE and LEO LOEB.

[From the Laboratory of Experimental Pathology, University of Pennsylvania. $]^{\mathrm{I}}$

I. The venom of Heloderma suspectum alone does not cause hemolysis of blood corpuscles (ox, sheep, dog, guinea-pig, rabbit, Heloderma, frog). Fresh and dried venom behave alike in this and other respects. It must be mentioned that on a few occasions guinea-pig corpuscles showed a trace of hemolysis with venom alone, but as this usually did not occur, it is likely that the corpuscles which behaved as exceptions, had, in some way, lost part of their natural resistance.

2. In combination with lecithin the venom causes hemolysis. The amount of lecithin necessary varies for corpuscles of different species.

3. Certain blood sera, heated or unheated, act like lecithin (dog, horse, turtle), others do not (guinea-pig, rabbit, ox, sheep, Heloderma). Only those activate which are supposed to act through lecithin, not those which act through complement.

4. A number of non-activating sera, heated or unheated, inhibit the venom-lecithin hemolysis. Different sera possess different inhibiting values, the greatest observed inhibition being due to Heloderma serum and the next greatest to guinea-pig serum. But the

${ }^{1}$ These experiments represent part of an investigation upon the toxic action of venom of Heloderma carried out in this laboratory under a grant from the Carnegie Institution. 